\title{
Genetic Incorporation of Olefin Cross-Metathesis Reaction Tags for Protein Modification
}

\author{
Bhaskar Bhushan, ${ }^{1}$ Yuya A. Lin, ${ }^{1 \dagger}$ Martin Bak, ${ }^{1}$ Anuchit Phanumartwiwath, ${ }^{1}$ Nan Yang, ${ }^{1}$ Matthew \\ K. Bilyard, ${ }^{1}$ Tomonari Tanaka, ${ }^{1} \zeta$ Kieran L. Hudson, ${ }^{1}$ Lukas Lercher, ${ }^{1}$ Monika Stegmann, ${ }^{2}$ Shabaz \\ Mohammed $^{1,2}$ and Benjamin G. Davis ${ }^{1, *}$ \\ ${ }^{1}$ Department of Chemistry, University of Oxford, Chemistry Res. Laboratory, Mansfield Rd, Oxford OXı 3TA UK \\ ${ }^{2}$ Department of Biochemistry, University of Oxford, OX1 3QU Oxford, UK \\ Supporting Information Placeholder
}

\begin{abstract}
Olefin cross-metathesis $(\mathrm{CM})$ is a viable reaction for the modification of alkene-containing proteins. Although allyl sulfide or selenide side-chain motifs in proteins can critically enhance the rate of CM reactions, no efficient method for their site-selective genetic incorporation into proteins has yet been reported. Here, through the systematic evaluation of olefin-bearing unnatural amino acids for their metabolic incorporation, we have discovered $S$-allylhomocysteine (Ahc) as a genetically encode-able Met analogue that is both processed by translational cellular machinery and is also a privileged CM substrate residue in proteins. In this way, Ahc was used for efficient Met-codon reassignment in a Met-auxotrophic strain of E. coli (B834 $\left.\left(D_{3}\right)\right)$, as well as metabolic labeling of protein in human cells and was reactive towards CM in several representative proteins. This expands the use of CM in the tool kit for 'tagand-modify' functionalization of proteins.
\end{abstract}

Alkenes can be installed into proteins by incorporation of some unnatural amino acids. ${ }^{1-4}$ However, although aliphatic alkene-containing amino acids such as homoallylglycine $(\mathrm{Hag})^{4}$ are reactive in both self-metathesis and crossmetathesis reactions as monomeric, protected amino acids in organic solvents ${ }^{5}$ and can be metabolically incorporated into proteins, they are unreactive in $\mathrm{CM}$ reactions in aqueous media. ${ }^{6}$ On the other hand, chemically-installed ${ }^{7}$ unnatural amino acids such as $S$-allylcysteine $(\mathrm{Sac})^{6}$ and $\mathrm{Se}$ allylselenocysteine $(\mathrm{Seac})^{8}$ have been demonstrated to be CM-reactive under aqueous conditions, with the allylic chalcogen heteroatom S (and even more so Se), providing crucial coordination to the metal catalyst centre. ${ }^{6,8,9}$ Schultz and co-workers have reported the incorporation of $O$-crotyl serine in yeast via amber stop-codon suppression, and have demonstrated its reactivity in on-protein intramolecular ring closing metathesis. ${ }^{10}$ However CM with geneticallyincorporated residues has yet to be demonstrated.

An alternative approach to 'non-sense' codon reassignment involves the direct commandeering of 'sense' codons for amino acids such as methionine (Met) and subsequent reassignment to incorporate unnatural amino acids (e.g. Hag, norleucine, trifluoromethionine, homopropargylglycine \& azidohomoalanine) as Met analogs. ${ }^{4,11-13}$ This effective reassignment of the Met codon exploits the flexibility of native methionyl-tRNA synthetase (MetRS) in accepting these analogs as substrates for tRNA loading. ${ }^{14}$ Here, by probing the flexibility of MetRS, we show metabolic labelling of proteins with an unnatural allyl chalcogen-containing amino acid (Met analogue $S$-allylhomocysteine (Ahc)) that is also metathesis-reactive.

The incorporation efficiency of amino acids and their analogs in vivo is controlled, to a significant extent, by their activation by the corresponding aminoacyl-tRNA synthetases (aaRS). ${ }^{4,15,16}$ Fersht \& Dingwall first demonstrated that a Met analogue, L-ethionine, may be mischarged by the MetRS without being metabolically edited. ${ }^{17}$ By contrast, Lhomocysteine, a natural competitor of Met, is transformed to the corresponding thiolactone to avoid misreading in translation. ${ }^{18}$ Side-chain length and heteroatom position can therefore be critical determinants in 'metabolic recognition' of such analogs. Therefore, in our design (Fig. 1) of putative analogs we explored these aspects: tolerance of MetRS towards positioning of the side-chain heteroatom required for CM plus concomitant variation of side-chain length.

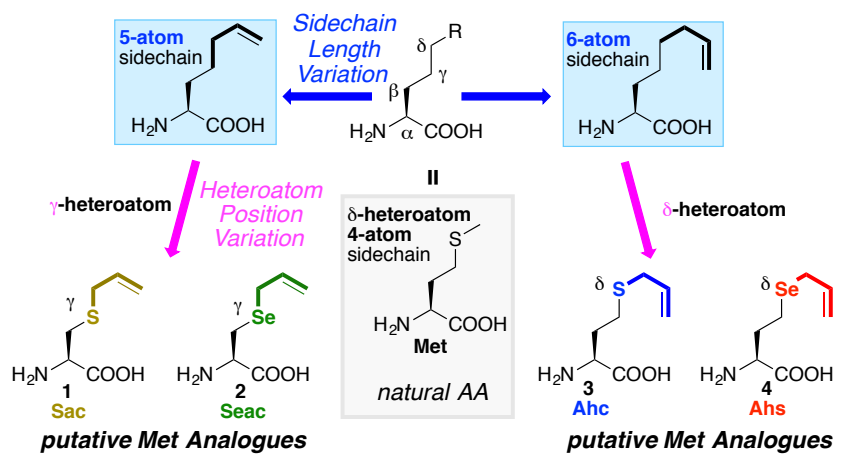

Figure 1. Design of $\gamma$-heteroatom Met analogs $S$ allylcysteine (Sac, 1), Se-allylselenocysteine (Seac, 2), and $\delta$-heteroatom analogs $S$-allylhomocysteine (Ahc, 3) \& Seallyl homoselenocysteine (Ahs, 4).

Two types of substrates were designed \& synthesized; those with a $\gamma$-heteroatom $(\mathbf{1}, \mathbf{2})$ and those with a $\delta$-heteroatom $(3$, 4). Previous work in our group has demonstrated that the chemical installation of Seac in proteins enhances the rate of 
on-protein CM and allows for an increased breadth of metathesis partners compared to Sac. ${ }^{8,9}$ Thus, the metabolic incorporation efficiencies of selenium analogues of 1 \& 3 (2 \& 4, respectively) were also considered. We reasoned too that since none of these uAAs possess a free side-chain $\mathrm{SH} / \mathrm{SeH}$ (unlike e.g. L-homocysteine), they would not be 'edited' through conversion to corresponding chalcogen lactones. 1,2 were prepared according to our prior methods. ${ }^{19} 3 \& 4$ were synthesized in homochiral form (L-3, L-4) from L-Met and LSeMet respectively using a demethylative allylation strategy that proved direct and efficient (Scheme 1). 3 could also be readily synthesized from the commercially available DLhomocysteine thiolactone as a racemate (DL-3, see SI).

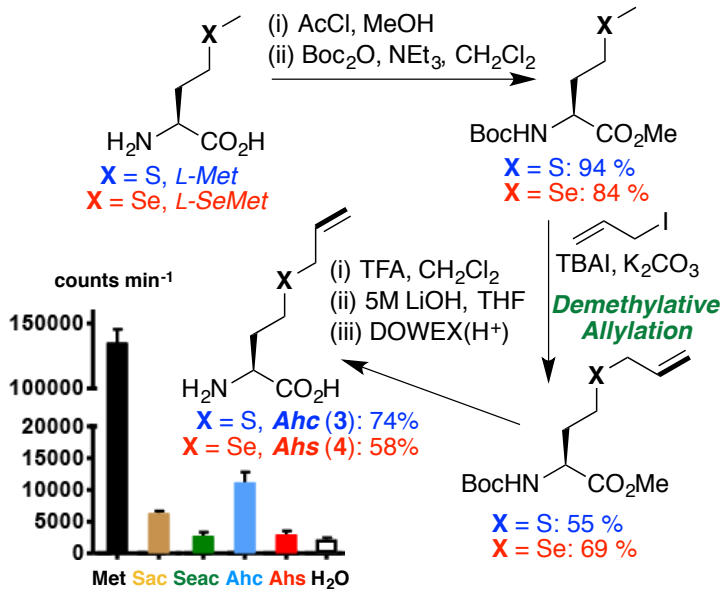

Scheme 1. Synthesis of $S$-allylhomocysteine (Ahc, 3) and Se-allylhomoselenocysteine (Ahs, 4) \& relative processing of Met and analogues 1-4 by MetRS determined through ATP-PPi exchange; endpoint 20 min (see SI).

First, to evaluate chalcogen-assisted CM reactivity of these motifs, protected forms of amino acids 1-4 were tested as small-molecule models (Scheme 2), as previously. ${ }^{6,8}$ Pleasingly, all proved CM-reactive towards allyl alcohol, under aq. conditions typical ${ }^{6-9}$ of prior successful protein $\mathrm{CM}$ reactions in reasonably short reaction times; little or no homodimerization was observed. Encouraged by these results, we next explored incorporation of 1-4 into proteins.

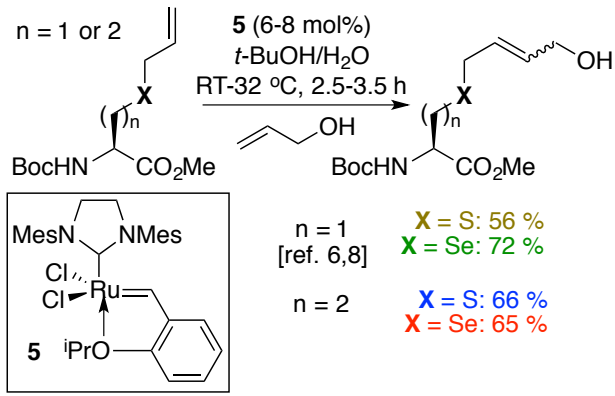

Scheme 2. Cross-metathesis on model amino acids Sac 1, Seac 2, Ahc 3 \& Ahs 4.

Molecular mechanics analysis was used to explore structural constraints in interactions with and processing by MetRS. Docking (see SI) of $\mathbf{1 - 4}$, and their corresponding adenylates, into the active site of $E$. coli MetRS (derived from Met-bound MetRS structure pdb ${ }_{1}$ PGo) used gradient-descent minimization of the energy of conformationally randomized ligands and an AMBER-derived forcefield (Figure 2 and SI). ${ }^{20}$
Pleasingly, in their minimized poses all UAA analogs were found to occupy the Met-binding site of MetRS (Figure $2 \mathrm{C}$ ). Consistent with previous studies, ${ }^{16}$ these revealed similar major contributors for binding of native Met (e.g. electrostatic interaction of Met- $\mathrm{N} \alpha$ with the sidechain of Asp52) to those known. Notably, in all cases (Met and uAAs 1-4) the heteroatom ( $\mathrm{S} / \mathrm{Se}$ ) of the sidechain was held by hydrogen bonding to the backbone amide $\mathrm{NH}$ of Leurz at the heart of the Met-binding site. However, flexibility in the hydrophobic 'end-wall' of the site (determined by Tyr26o), led to accommodation of a range of sidechain termini (Me or allyl, Fig. 2): heteroatom-to-Tyr26o-O $\omega$ distances were displace by up to $\sim 0.5 \AA$. Moreover, primary binding contact with Asp52 was lost or distorted. Notably, for Ahc (3) compensatory interactions were predicted between Ahc$\mathrm{C} \alpha(=\mathrm{O}) \mathrm{O}$ and both of the backbone amide NHs of Tyr15 \& Pro14 (Fig. 2B). Together these data highlighted both the critical role of sidechain heteroatom positioning and encouragingly suggested sufficient flexibility of MetRS (in accommodating extended termini Me / allyl) as well as compensatory binding modes in certain cases (i.e. Ahc).

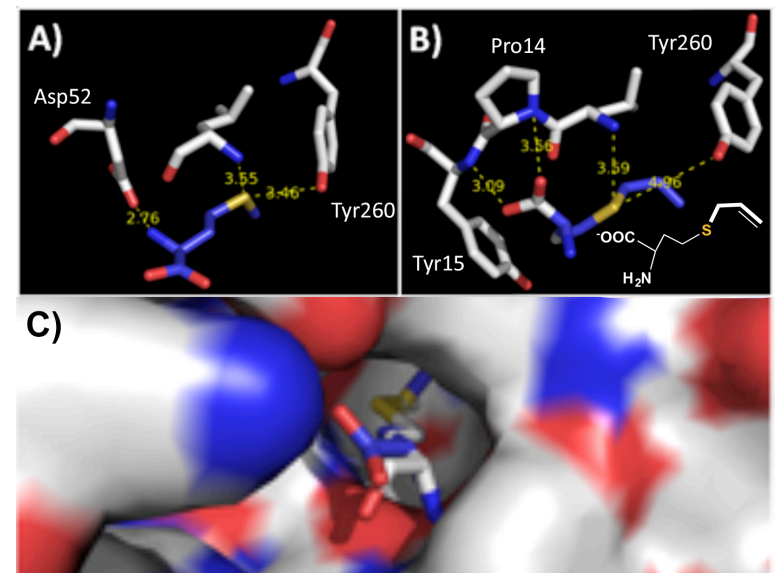

Figure 2. Interactions of docked amino acids with MetRS for A) Met, B) Ahc (see Fig S1 for further poses of other analogs 1-4). C) Docked Met (blue backbone) adopts a similar pose in the same Met pocket of MetRS identified by crystallography (pdb 1 PGo, white backbone).

To experimentally test these predictions, E. coli MetRS was expressed $^{19}$ and the relative activities for loading of Met, and analogs 1-4 onto tRNA determined using ATP-PPi exchange (see Scheme 1 and SI). ${ }^{21}$ Met, as natural substrate, proved most effective, as expected. However, from the panel of olefinic uAAs 1-4, only the $S$-allyl uAAs 1 \& 3 showed significant activity above background; Ahc 3 showed the greatest activity ( $\sim 6$-fold $>1$, Scheme 1$)$. Determination and comparison of the Michaelis-Menten parameters for Met \& 3 (Table 1, SI and Fig S2) suggests that both display similar binding to MetRS (as judged by $K_{\mathrm{M}}$ ) although 3 has a lower $\mathrm{k}_{\mathrm{cat}}$. Thus, despite the clear differences in structure, the turnover (as judged by $\mathrm{k}_{\mathrm{cat}} / K_{\mathrm{M}}$ ) of Ahc (3) by MetRS was less than an order-of-magnitude lower than for native substrate Met (Table 1). Following these in silico \& in vitro predictions \& validations of putative $\mathrm{uAA}$ processing, in vivo incorporation of each Met analog was investigated. The nucleosome constituent protein histone $\mathrm{H}_{3}{ }^{22,23}$ was tested as the first target model protein using the Met-auxotrophic B834(DE3) E. coli strain. Efficient incorporation (> 95\%) was 
only observed for Ahc 3, by both LCMS and tryptic digestMSMS (Fig. 3 and SI).

Table 1. Michaelis-Menten Parameters for MetRS

\begin{tabular}{ccc}
\hline & Met & Ahc (3) \\
$\mathrm{k}_{\mathrm{cat}}\left(\mathrm{s}^{-1}\right)$ & $23.2 \pm 2.7$ & $3.1 \pm 2.7$ \\
$K_{\mathrm{M}}(\mu \mathrm{M})$ & $870 \pm 188$ & $1030 \pm 235$ \\
$\mathrm{k}_{\mathrm{cat}} / K_{\mathrm{M}}\left(\mathrm{s}^{-1} \mu \mathrm{M}^{-1}\right)$ & $0.026 \pm 0.006$ & $0.0030 \pm 0.0007$ \\
\hline
\end{tabular}

${ }^{a}$ See also Figure 3 B,C and SI for further details.

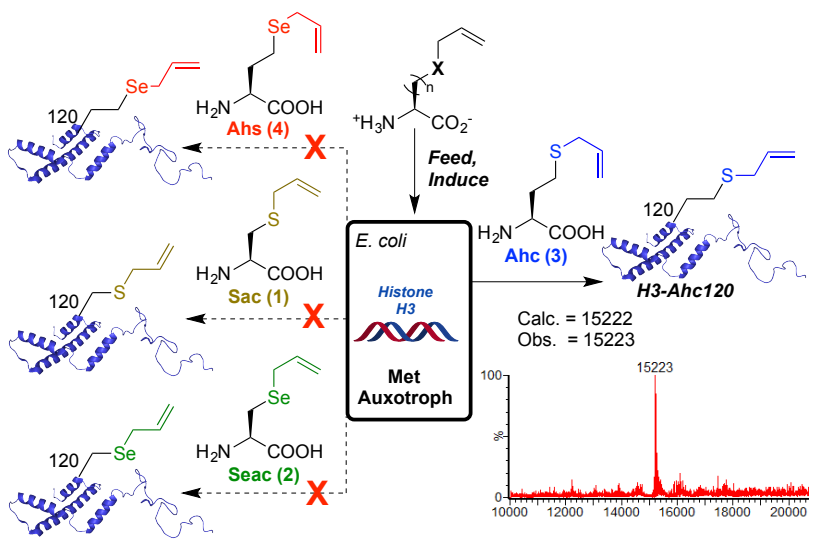

Figure 3. Successful incorporation of Ahc, 3 into Histone $\mathrm{H}_{3}$; Sac 1, Seac 2 and Ahs 4 were not incorporated (see SI).

These experiments highlighted 3 as the most suitable for genetic incorporation. Proteins of varying structure and function were chosen to test scope. Thus, in addition to $\mathrm{H}_{3}$, single-site incorporations of Ahc were successfully carried out in right-handed $\beta$-helix pentapeptide repeat protein $\mathrm{Np}_{276,}{ }^{24}$ and in TIM-barrel $\beta$-glycosidase Ss $\beta \mathrm{G}^{25}$ Excellent levels $(>95 \%)$ of multi-site incorporation of Ahc were also demonstrated: in monomeric $\alpha$-helix bundle DNA-binding protein SarZ, ${ }^{26}$ as well as multimeric bacteriophage coat protein $Q \beta .^{27}$ For all proteins, site and level of incorporation was confirmed by LCMS of intact protein and tryptic-MSMS analysis. Ahc-incorporated proteins retained both secondary \& tertiary structure (as determined by $C D$, see $\mathrm{SI}$ ) and function (retention of glycosidase activity by $\mathrm{Ss} \beta \mathrm{G}$, and DNA binding by SarZ, see SI and Figs S6-8). Typical yields (mg/L) for Ahc-incorporated proteins were: SarZ $\sim 0.5$ (in LBQB), $\sim 2.9$ (LB); Ss $\beta \mathrm{G} \sim 4.5$ (LB); IgG-Fc 1 (DMEM); H3 2.4 (LB); $\mathrm{Np} 276 \sim 1.1$ (LB).

With Ahc-containing proteins in hand, their reactivity towards CM was tested; allyl alcohol or fluorescein-olefin 6 were used as model metathesis partners. Evaluations of reaction conditions and optimizations were conducted through systematic variation of various parameters (SI Tables $\mathrm{S}_{1}$ and $\mathrm{S}_{2}$ ) and highlighted: the utility of $t \mathrm{BuOH}$ or DMSO as co-solvents (where needed) lacking alpha-protons; potential utility of PEG-500 as co-solvent; strong benefit of $\mathrm{MgCl}_{2}$ as an additive; ${ }^{6}$ phosphate-based buffers and mild denaturant (e.g. through guanidinium) to increase solubility and stability; and $\mathrm{Ru}$ scavenging during work-up for LCMS monitoring. Following this process and, consistent with the observed reactivity for Ahc in model amino acids, CM was observed on all tested Ahc-containing proteins with conversions ranging from $55 \%$ (for bulkier 6) to $>95 \%$ for allyl alcohol, including for the intact $Q \beta$ virus-like particle bearing 180 reaction sites (Scheme 3), and for SarZ, which has two reaction sites. $S s \beta G$ was found to retain its functional activity following CM reactions (see Table $\mathrm{S}_{4}$ ).

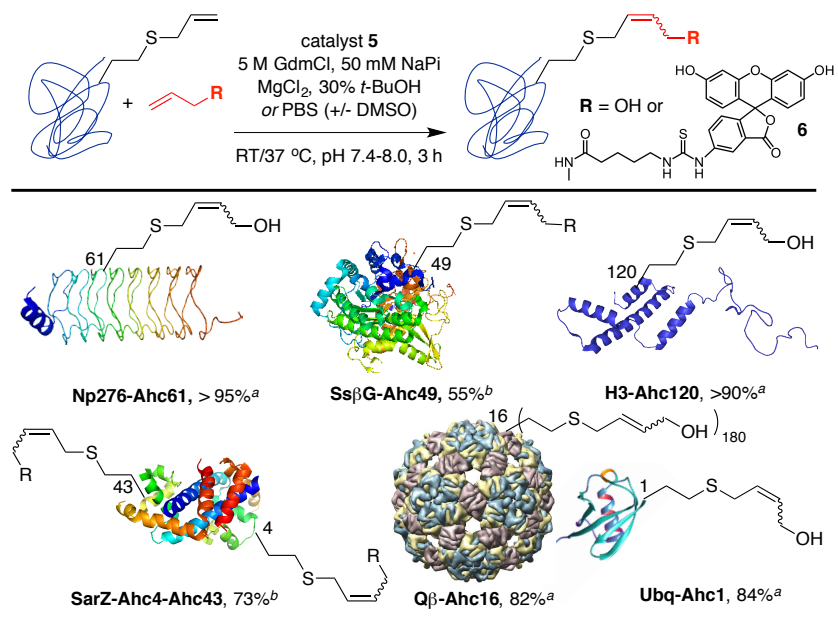

Scheme 3. CM on Ahc-incorporated proteins: single-site Np276, Histone $\mathrm{H}_{3}, \mathrm{Ss} \beta \mathrm{G}$, Ubq and multi-site incorporated SarZ and Q $\beta$. Conversion based on ${ }^{a} \mathrm{MS}$ with allyl alcohol, or ${ }^{b}$ fluorescence assay (Figs $\mathrm{S}_{3}-5$ ) with 6.

Finally, direct Ahc incorporation in mammalian cells could have useful application in the generation of probes in cellulo and in chemical proteomic strategies (such as noncanonical amino acid tagging ${ }^{28,29}$ ). We next tested and demonstrated such incorporation using Ahc in human (HEK293T) cells (Fig. 4 \& SI). As a test protein from human cells, not only was installation of Ahc into the Fc region of IgG determined by both MS \& MSMS (see SI) but the incorporated Ahc was also found to be reactive, allowing direct CM-labeling of IgG-Fc through reaction with olefinic-biotin $7 \cdot^{30} 7$ also proved effective in reaction with other proteins (e.g. SarZ, Ss $\beta$ G, see SI). Given the potential used of biotin tags in affinity proteomic methods, ${ }^{28,29}$ this both highlights the capability of Ahc to serve as a general Met analogue across different translational systems / cell types and also suggests its utility in the future interrogation of human proteomes.

In conclusion, our results demonstrate that the previously unexplored amino acid $S$-allyl homocysteine (Ahc) is an effective Met surrogate that is incorporated into proteins not only by the translational apparatus of Met-auxotrophic E. coli (with good efficiency $>95 \%$ ) thereby allowing genetic control of olefin cross metathesis in proteins using sense (Met) codon reassignment but also even into human cells. Such incorporation could be potentially be improved further by use of MetRS variants. ${ }^{3}$ It should be noted that there remain some limitations of the CM method; we observed here, for example, that in one case the use of $\mathrm{tBuOH}$ for improving the solubility of catalyst 5 led to the loss of activity of the highly solvent-sensitive protein SarZ (see SI). In addition, whilst reactions using $\mathbf{5}$ typically proceed with strong $E$ selectivity, ${ }^{9}$ some E/Z-heterogeneity may also exist in conjugates. Thus, with the continuing development of watersoluble $^{31,32}$ or Z-selective ${ }^{33}$ catalysts for cross-metathesis and the genetically-controlled capabilities disclosed here, there is now good motivation for the development of effective, in vivo Ahc-enabled CM in cellular systems, thereby enabling access to a broad range of metal-mediated biological applications. 


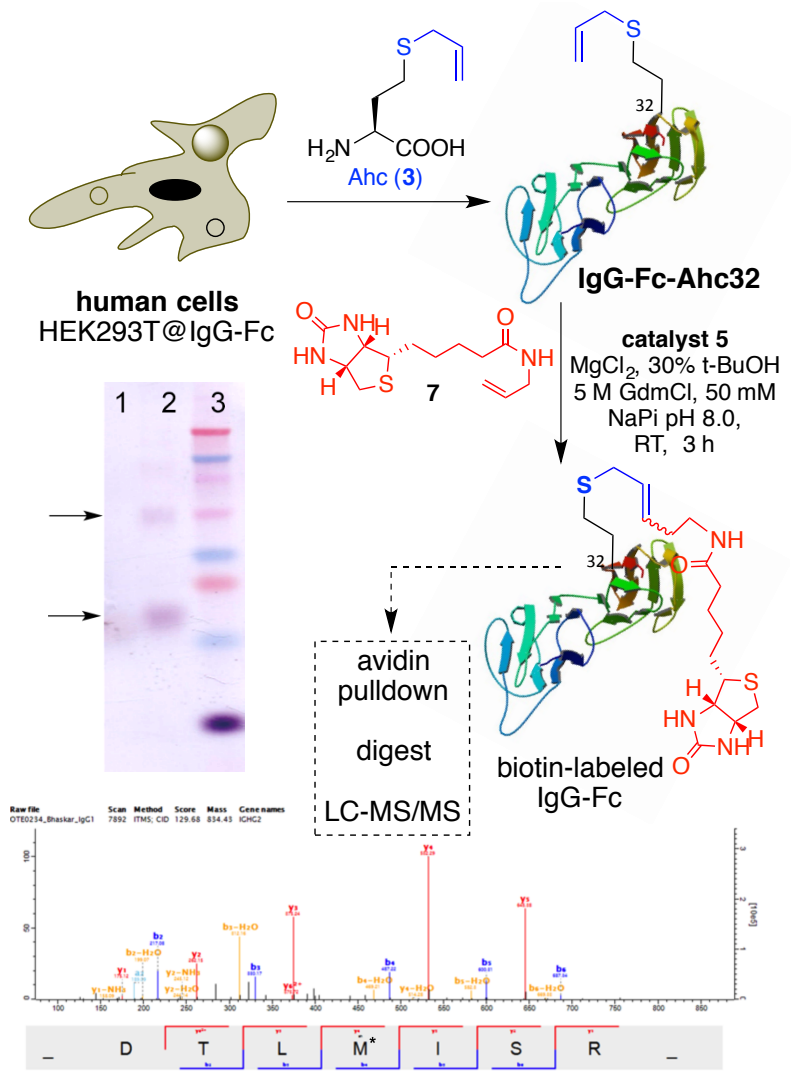

Figure 4. Use of Ahc in human cells and CM-labelling of Ahc-incorporated IgG-Fc with olefin-biotin 7. Insets show MS/MS of isolated $\mathrm{IgG}_{-} \mathrm{FC}_{29-36}$ peptide $\mathrm{DTL}-\mathrm{M}^{*}$-IMSR containing analog $\mathrm{M}^{*}=3$ and anti-biotin Western (nonreducing, size markers, 20, 30, 40, 50, 60, 80, $110 \mathrm{kDa}$; arrows show IgG-Fc monomer and dimer) demonstrating incorporation and $\mathrm{CM}$ reaction with 7 , respectively. Dotted box shows potential affinity proteomic workflow.

\section{ASSOCIATED CONTENT}

\section{Supporting Information}

Full procedures and protein ESI-MS. Material is available free of charge via the Internet at http://pubs.acs.org.

\section{Corresponding Author}

ben.davis@chem.ox.ac.uk

\section{Present Addresses}

${ }^{\dagger}$ Department of Chemistry, National Sun Yat-sen University, No. 70, Lienhai Rd, Kaohsiung, Taiwan $\xi$ Department of Biobased Materials Science, Kyoto Institute of Technology, Matsugasaki, Sakyo-ku, Kyoto, Japan.

\section{Notes}

The authors declare no competing financial interests.

\section{ACKNOWLEDGMENT}

We thank the Rhodes Trust (BB), EPA Cephalosporin Scholarship (BB), EPSRC (YAL, LL) \& CRUK (LL); Royal Society Wolfson Research Merit Award (BGD) for funding; Florian Seebeck \& Ritu Raj for discussions; and James Wickens, Sonia de Munari for technical assistance.
(1) van Hest, J. C. M.; Tirrell, D. A. Efficient introduction of alkene functionality into proteins in vivo. FEBS Lett. 1998, 428 , 68.

(2) Song, W.; Wang, Y.; Yu, Z.; Vera, C. I. R.; Qu, J.; Lin, Q. A Metabolic Alkene Reporter for Spatiotemporally Controlled Imaging of Newly Synthesized Proteins in Mammalian Cells. ACS Chem. Biol. 2010, 5, 875 .

(3) Ngo, J. T.; Tirrell, D. A. Noncanonical amino acids in the interrogation of cellular protein synthesis. Acc. Chem. Res. 2011, 44,677 .

(4) van Hest, J. C. M.; Kiick, K. L.; Tirrell, D. A. Efficient Incorporation of Unsaturated Methionine Analogues into Proteins in Vivo. J. Am. Chem. Soc. 2000, 122, 1282.

(5) Gibson, S. E.; Gibson, V. C.; Keen, S. P. Cross metathesis of the amino acid homoallylglycine. Chem. Commun. 1997, 1107.

(6) Lin, Y. A.; Chalker, J. M.; Floyd, N.; Bernardes, G. J.; Davis, B. G. Allyl sulfides are privileged substrates in aqueous crossmetathesis: application to site-selective protein modification. $J$. Am. Chem. Soc. 2008, 130, 9642.

(7) Chalker, J. M.; Lin, Y. A.; Boutureira, O.; Davis, B. G. Enabling olefin metathesis on proteins: chemical methods for installation of S-allyl cysteine. Chem Commun 2009, 3714.

(8) Lin, Y. A.; Boutureira, O.; Lercher, L.; Bhushan, B.; Paton, R. S.; Davis, B. G. Rapid cross-metathesis for reversible protein modifications via chemical access to Se-allyl-selenocysteine in proteins. J. Am. Chem. Soc. 2013, 135, 12156.

(9) Lin, Y. A.; Chalker, J. M.; Davis, B. G. Olefin cross-metathesis on proteins: investigation of allylic chalcogen effects and guiding principles in metathesis partner selection. J. Am. Chem. Soc. 2010, 132, 16805 .

(10) Ai, H. W.; Shen, W.; Brustad, E.; Schultz, P. G. Genetically encoded alkenes in yeast. Angew. Chem. Int. Ed. 2o10, 49, 935.

(11) Kiick, K. L.; Saxon, E.; Tirrell, D. A.; Bertozzi, C. R. Incorporation of azides into recombinant proteins for chemoselective modification by the Staudinger ligation. Proc. Natl. Acad. Sci. USA 2002, 99, 19.

(12) Duewel, H.; Daub, E.; Robinson, V.; Honek, J. F. Incorporation of trifluoromethionine into a phage lysozyme: implications and a new marker for use in protein ${ }_{19} \mathrm{~F}$ NMR. Biochemistry 1997, 36, 3404.

(13) Bogosian, G.; Violand, B. N.; Dorwardking, E. J.; Workman, W. E.; Jung, P. E.; Kane, J. F. Biosynthesis and Incorporation into Protein of Norleucine by Escherichia-Coli. J Biol Chem 1989, 264 , 531.

(14) Budisa, N. Prolegomena to future experimental efforts on genetic code engineering by expanding its amino acid repertoire. Angew Chem Int Ed Engl 2004, 43, 6426.

(15) Hartman, M. C.; Josephson, K.; Lin, C. W.; Szostak, J. W. An expanded set of amino acid analogs for the ribosomal translation of unnatural peptides. PLoS One $\mathbf{2 0 0 7}, 2$, e972.

(16) Datta, D.; Vaidehi, N.; Zhang, D.; Goddard, W. A. Selectivity and specificity of substrate binding in methionyl-tRNA synthetase. Protein Sci. 2004, 13, 2693.

(17) Fersht, A. R.; Dingwall, C. An editing mechanism for the methionyl-tRNA synthetase in the selection of amino acids in protein synthesis. Biochemistry 1979, 18, 1250.

(18) Jakubowski, H. Proofreading in vivo: editing of homocysteine by methionyl-tRNA synthetase in Escherichia coli. Proc. Natl. Acad. Sci. USA 1990, 87, 4504.

(19) Shimizu, Y.; Inoue, A.; Tomari, Y.; Suzuki, T.; Yokogawa, T.; Nishikawa, K.; Ueda, T. Cell-free translation reconstituted with purified components. Nat. Biotech. 2001, 19, 751.

(20) Morris, G. M.; Huey, R.; Lindstrom, W.; Sanner, M. F.; Belew, R. K.; Goodsell, D. S.; Olson, A. J. AutoDock4 and AutoDockTools4: Automated Docking with Selective Receptor Flexibility. J. Comp. Chem. 2009, 30, 2785.

\section{REFERENCES}


(21) Ghosh, G.; Pelka, H.; Schulman, L. H. Identification of the tRNA anticodon recognition site of Escherichia coli methionyltRNA synthetase. Biochemistry 1990, 29, 2220.

(22) Rea, S.; Eisenhaber, F.; O'Carroll, D.; Strahl, B. D.; Sun, Z.W.; Schmid, M.; Opravil, S.; Mechtler, K.; Ponting, C. P.; Allis, C.

D.; Jenuwein, T. Regulation of chromatin structure by sitespecific histone $\mathrm{H}_{3}$ methyltransferases. Nature 2ooo, 406, 593.

(23) Kouzarides, T. Chromatin Modifications and Their Function. Cell 2007, 128, 693.

(24) Vetting, M. W.; Hegde, S. S.; Hazleton, K. Z.; Blanchard, J. S. Structural characterization of the fusion of two pentapeptide repeat proteins, $\mathrm{Np2} 75$ and Np276, from Nostoc punctiforme: Resurrection of an ancestral protein. Protein Science 2007, 16, 755 .

(25) Aguilar, C. F.; Sanderson, I.; Moracci, M.; Ciaramella, M.; Nucci, R.; Rossi, M.; Pearl, L. H. Crystal structure of the $\beta$ glycosidase from the hyperthermophilic archeon Sulfolobus solfataricus: resilience as a key factor in thermostability. J. Mol. Biol. 1997, 271, 789.

(26) Kaito, C.; Morishita, D.; Matsumoto, Y.; Kurokawa, K.; Sekimizu, K. Novel DNA binding protein SarZ contributes to virulence in Staphylococcus aureus. Molec. Microbiol. 20o6, 62, 1601.

(27) Kozlovska, T. M.; Cielēns, I.; Dreilinņa, D.; Dišlers, A.; Baumanis, V.; Ose, V.; Pumpēns, P. Recombinant rna phage q $\beta$ capsid particles synthesized and self-assembled in Escherichia coli. Gene 1993, 137, 133.

(28) Dieterich, D. C.; Link, A. J.; Graumann, J.; Tirrell, D. A.; Schuman, E. M. Selective identification of newly synthesized proteins in mammalian cells using bioorthogonal noncanonical amino acid tagging (BONCAT). Proc. Natl. Acad. Sci. USA 2006, 103, 9482.

(29) Howden, A. J. M.; Geoghegan, V.; Katsch, K.; Efstathiou, G.; Bhushan, B.; Boutureira, O.; Thomas, B.; Trudgian, D. C.; Kessler, B. M.; Dieterich, D. C.; Davis, B. G.; Acuto, O. QuaNCAT: quantitating proteome dynamics in primary cells. Nat. Methods 2013, 10, 343.

(30) Wittrock, S.; Becker, T.; Kunz, H. Synthetic Vaccines of Tumor-Associated Glycopeptide Antigens by ImmuneCompatible Thioether Linkage to Bovine Serum Albumin. Angew Chem Int Ed Engl 2007, 46, 5226.

(31) Hong, S. H.; Grubbs, R. H. Highly Active Water-Soluble Olefin Metathesis Catalyst. J. Am. Chem. Soc. 2006, 128, 3508.

(32) Skowerski, K.; Szczepaniak, G.; Wierzbicka, C.; Gulajski, L.; Bieniek, M.; Grela, K. Highly active catalysts for olefin metathesis in water. Catal Sci Technol 2012, 2, 2424.

(33) Herbert, M. B.; Grubbs, R. H. Z-Selective Cross Metathesis with Ruthenium Catalysts: Synthetic Applications and Mechanistic Implications. Angew Chem Int Ed Engl 2015, 54, 5018 . 
For Table of Contents

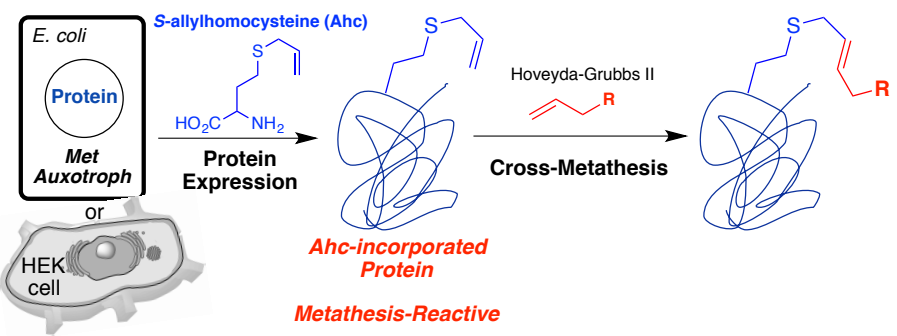

